\title{
High Crystal Quality 2D Manganese Phosphorus Trichalcogenide Nanosheets and their Photocatalytic Activity
}

\author{
Tofik Ahmed Shifa, Fengmei Wang, Zhongzhou Cheng, Peng He, Yang Liu, Chao Jiang, \\ Zhenxing Wang, * and Jun He*
}

Transition metal phosphorus trichalcogenides $\left(\mathrm{MPX}_{3}, \mathrm{X}=\mathrm{S}\right.$, Se) are layered materials possessing high chemical diversity and wide range of applications in a broad wave length spectrum. Theoretical studies reveal that auspicious activity of photocatalytic water splitting can be realized from them. However, experimental efforts have so far been challenged with the synthesis bottleneck. Described herein is the general chemical vapor deposition (CVD) growth method and photocatalytic activity of these materials. A novel route to systematically grow $\mathrm{MnPX}_{3}$ nanosheets on flexible carbon fiber substrate is reported. The temperature profile of the CVD process is carefully optimized that confer a facile and successful conversion of oxide precursor to phosphotrichalcogenide with high crystallinity. Moreover, the obtained manganesebased phosphorus trichalcogenide nanosheets demonstrate promising activity in sacrificial agent-free photocatalytic water splitting under simulated solar light (AM 1.5G). This study provides a significant stepping stone in exploring the fascinating world of functional 2D materials and pursuing performance enhancement.

\section{Introduction}

The production of hydrogen molecule through water splitting has remained a significant issue of investigation in connection to the effort of sustainably powering the globe. ${ }^{[1]}$ Considering the

T. A. Shifa, Dr. F. M. Wang, Z. Cheng, P. He, Y. Liu, Prof. Z. Wang, Prof. J. He

CAS Center for Excellence in Nanoscience

CAS Key Laboratory of Nanosystem and Hierarchical Fabrication

National Center for Nanoscience and Technology

Beijing 100190, China

E-mail:wangzx@nanoctr.cn; hej@nanoctr.cn

T. A. Shifa, Y. Liu, Prof. J. He

University of Chinese Academy of Sciences

Beijing 100049, China

Z. Cheng

School of Materials Science and Engineering

University of Science and Technology Beijing

Beijing 100083, China

Prof. C. Jiang

CAS Key Laboratory of Standardization and Measurement for Nanotechnology

National Center for Nanoscience and Technology

Beijing 100190, China

The ORCID identification number(s) for the author(s) of this article can be found under https://doi.org/10.1002/adfm.201800548.

DOI: $10.1002 / \mathrm{adfm} .201800548$ effective charge/electron transport, appreciable stability, and immensely exposed active surface sites, 2D layered transition metal dichalcogenides $\left(\mathrm{MX}_{2}\right)$ have generated a tremendous amount of interest in catalyzing the dissociation of water into its component parts. ${ }^{[2]}$ Fascinating strategies have been communicated so far aiming at their controllable synthesis and performance enhancement. ${ }^{[3]}$ In catalyzing hydrogen evolution reaction (HER), the crucial role of foreign $\mathrm{P}$ site in $\mathrm{MX}_{2}$ is greatly appreciated as evidenced in $\mathrm{MoS}_{2},{ }^{[4]} \mathrm{WS}_{2}{ }^{[5]}$ and $\mathrm{CoS}_{2}{ }^{[6]}$ The extraordinarily high activities exhibited by CoPS, ${ }^{[7]} \mathrm{CoS} / \mathrm{P}^{[8]} \mathrm{MoP} / \mathrm{S}^{[9]}$ shine more light on this postulate. Moreover, it has been pragmatic that increasing the atomic content of $\mathrm{P}$ in transition metal phosphide ${ }^{[10]}$ such as, $\mathrm{Ni}_{x} \mathrm{P}_{y},{ }^{[11]} \mathrm{Mo}_{x} \mathrm{P}_{Y},{ }^{[12]}$ and $\mathrm{Co}_{x} \mathrm{P}_{y},{ }^{[13]}$ causes the increased HER activity. Therefore, the presence of a transition metal, chalcogen, phosphorus, and the $2 \mathrm{D}$ feature as integral part of the catalyst can bring about the realization of excellent catalysis. In this aspect, transition metal phosphorus trichalcogenides $\left(\mathrm{MPX}_{3}\right)$ are the best candidates. $\mathrm{MPX}_{3}$ is one of the families of layered 2D materials whose electronic and structural features provide appealing etiquettes for catalysis. ${ }^{[14]}$ This family demonstrates higher chemical diversity and structural complexity as compared to the conventional layered $\mathrm{MX}_{2}$ thereby opening a wide range of applications in a broad wave length spectrum. ${ }^{[15]}$ Despite its long history (beginning in the 19th century) ${ }^{[16]}$ of main scientific direction, the development of $\mathrm{MPX}_{3}$ for catalysis is still at its infant stage. This is associated with the difficulties in controllably synthesizing nanoscaled $\mathrm{MPX}_{3}$ crystals. The methods employed so far such as chemical vapor transport (CVT) require quite long time of reaction up to couple of days, weeks or even months. ${ }^{[17]}$ It involves a mere reaction of stoichiometric amounts of the components (metal, chalcogen, and phosphorus) in evacuated tube. The resultant $\mathrm{MPX}_{3}$ is generally big in size and low in specific surface area to volume ratio. Therefore, the CVT method is not a practicable way to control the morphology of $\mathrm{MPX}_{3}$ and hence thorough investigation of this family in various applications has been thwarted due to the synthesis bottleneck. Serendipitously, chemical vapor deposition (CVD) is well acknowledged route for swiftly growing various $\mathrm{MX}_{2}$ nanostructures with enormously exposed active sites for catalysis. ${ }^{[18]}$ Unlike $\mathrm{MX}_{2}$, the growth of $\mathrm{MPX}_{3}$ via this method requires a careful optimization of temperature as the group- $\mathrm{V}$ 
atom, $\mathrm{P}$, has to be vaporized along with the chalcogen atom, $\mathrm{X}$, and completely react with the metal oxide precursor. Our group has recently reported the photocatalytic hydrogen evolving activity of ultrathin $\mathrm{NiPS}_{3}$ nanosheet which uncovers the promising feature of this family toward water splitting. ${ }^{[19]}$ Herein, we further introduce a "preheating" process into the CVD growth to confirm the formation of fused mixture of $\mathrm{P}$ and $\mathrm{X}$ atoms before they are carried to the back zone for reacting with the metal oxide precursor. Being a theoretically proved promising photocatalyst with direct band gap, ${ }^{[20]}$ manganese-based transition metal phosphorus trichalcogenides $\left(\mathrm{MnPX}_{3}\right)$ are chosen and synthesized with our developed method. This novel route resulted in a successful synthesis of monoclinic $\mathrm{MnPS}_{3}$ and hexagonal $\mathrm{MnPSe}_{3}$ nanosheets with high crystalline quality and phase purity. Their band gaps and the phase structure were studied in detail. Notably, their valance band (VB) and conduction band (CB) energy levels are constituted from the $\mathrm{P}_{2} \mathrm{X}_{6}{ }^{4-}$ cluster orbitals. Going from $\mathrm{P}_{2} \mathrm{~S}_{6}{ }^{4-}$ to $\mathrm{P}_{2} \mathrm{Se}_{6}{ }^{4-}$, the electronic structure and the nature of energy levels are altered following the difference between $\mathrm{S}$ and Se atoms. The light absorption property shows an obvious red shift for $\mathrm{MnPSe}_{3}$. Interestingly, the synthesized nanosheets demonstrated unassisted photocatalysis for hydrogen generation in sacrificial agent-free water. The hydrogen evolution rate is about $6.5 \mu \mathrm{mol} \mathrm{g}{ }^{-1}$ for $\mathrm{MnPSe}_{3}$ nanosheets, which shows a better performance than that of $\mathrm{MnPS}_{3}$ nanosheets $\left(3.1 \mu \mathrm{mol} \mathrm{g}{ }^{-1}\right)$. Being the first experimental report regarding the catalytic property of facilely synthesized $\mathrm{MnPX}_{3}$, our finding can be an essential stepping stone in pursuit of performance enhancement and further exploration of other 2D materials in this emerging family.

\section{Results and Discussion}

We followed a systematic two step method (see the Experimental Section) which is a novel route for obtaining $\mathrm{MnPX}_{3}$ at the level of nanometer thickness. First, $\mathrm{MnO}_{2}$ nanosheet (NS) was grown hydrothermally on preacidified carbon fiber (CF) substrate. The morphology, as obtained from scanning electron microscope and transmission electron microscope (TEM), the $\mathrm{X}$-ray diffraction (XRD) pattern and high resolution TEM image are illustrated in Figures S1 and S2 (Supporting Information). It is obvious that the nanosheet grew vertically covering the entire surface of the substrate with crystallographic pattern matching to tetragonal $\mathrm{MnO}_{2}$ (PDF\#72-1982). In the second step, the obtained $\mathrm{MnO}_{2} \mathrm{NS} / \mathrm{CF}$ was placed at the back zone of CVD furnace wherein the front zone was loaded by a mixture of $\mathrm{P}$ and $\mathrm{S} / \mathrm{Se}$ (accordingly) powders. Two growth routes were conducted (Figure 1). In route-I, we tried direct heating of the front zone along with the back zone simultaneously, which is commonly used in the CVD synthesis of $\mathrm{MX}_{2}$ nanomaterials. ${ }^{[18]}$ Nonetheless, prior selenization occurred inhibiting the pure phase synthesis of $\mathrm{MnPSe}_{3}$. According to the XRD pattern (Figure 1b), both cubic MnSe and hexagonal $\mathrm{MnPSe}_{3}$ coexisted in the grown material. Thus, initial creation of thiophosphate/selenophosphate atmosphere (route-II, Figure 1) in the CVD system was found vividly crucial to successfully get the desired $\mathrm{MnPX}_{3}$ phase. For this, the front zone was preheated at $110{ }^{\circ} \mathrm{C}$ for $10 \mathrm{~min}$ to make a fused mixture of phosphorus and chalcogen atoms that lead to a phase pure product. This phase purity and the crystalline structure of the as-grown materials were ascertained from XRD analysis. It is depicted in the XRD pattern of route-II that the peaks for $\mathrm{MnPSe}_{3}$ matches well to standard diffractogram of hexagonal crystalline structure (PDF\# 89-1414) and that of $\mathrm{MnPS}_{3}$ (Figure S3, Supporting Information) appears in monoclinic arrangement (PDF\#78-0495). A typical metal phospho-trichalcogenide crystal can be regarded as a composition of two cations $\left(\mathrm{M}^{2+}\right)$ and one $\left[\mathrm{P}_{2} \mathrm{X}_{6}\right]^{4-}$ anion cluster in which $2 / 3$ of the octahedral centers is occupied by $\mathrm{M}^{2+}$. The P-P dimers that are covalently bonded to chalcogen with a tetrahedral like arrangement (3X atoms with a)

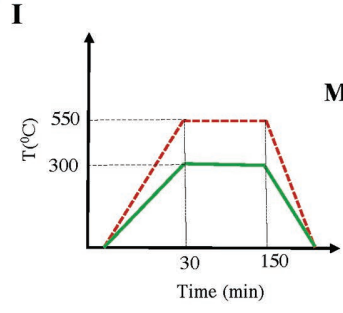

\section{Mixed Phases}
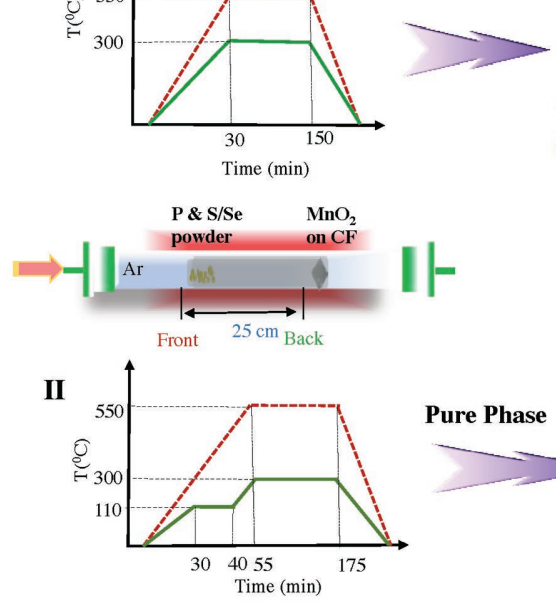

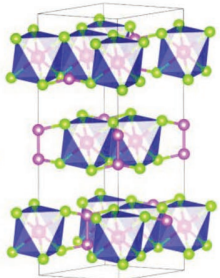

$\mathrm{MnPSe}_{3}$, Hexagonal

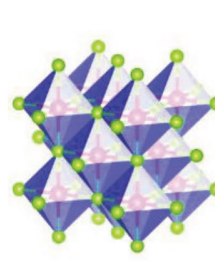

MnSe, Cubic

b)

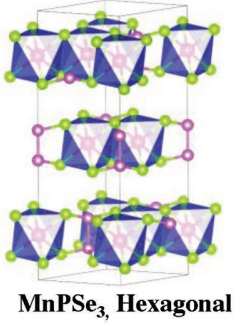

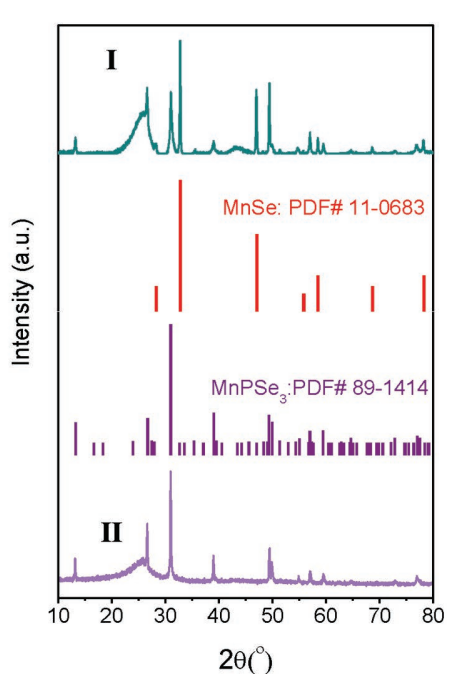

Figure 1. a) Temperature profiles of chemical vapor deposition routes leading to mixed phase (route-I) and pure phase (route-II) products along with schematic of their chemical structures. b) XRD patterns of the grown materials through both routes. 


\section{ADVANCED}

each $\mathrm{P}$ atom) occupy the remaining $1 / 3$ part. They are layered structures having a weak van der Waals interaction between the layers and a $\mathrm{P}_{2} \mathrm{X}_{6}$ anion unit within each lamella. In $\mathrm{MnPSe}_{3}$, the atomic layers stack in R3 space group such that each Se atom is bonded to single $\mathrm{P}$ atom and coordinated by two $\mathrm{Mn}$ sites in hexagonal arrangement (Figure S3b, Supporting Information). Coming to $\mathrm{MnPS}_{3}$, structural difference arises owing to the decreased bond distance $(\mathrm{P}-\mathrm{S})$ and bond angles $(\mathrm{S}-\mathrm{P}-\mathrm{S})$ as compared to its selenide equivalent. The atomic layers stack in the $\mathrm{C} 2 / \mathrm{m}$ space group of monoclinic structure (Figure S3b, Supporting Information). As can be seen from the images of scanning electron microscopy (Figure S4, Supporting Information), their morphology resembles hexagonal nanosheets grown on CF. The nanosheets cover the surface of the CFs uniformly. For a clearer visualization using atomic force microscopy (AFM), we transferred the sample from CF to $\mathrm{SiO}_{2} / \mathrm{Si}$ substrate and the images are shown in Figure 2a,b. It is evident that the transferred nanosheets are as thin as $\approx 6 \mathrm{~nm}$ and $\approx 28 \mathrm{~nm}$ with lateral size of 1.5 and $0.45 \mu \mathrm{m}$ for $\mathrm{MnPS}_{3}$ and $\mathrm{MnPSe}_{3}$, respectively. Moreover, energy dispersive X-ray spectroscopic (EDX)-elemental analysis confirms homogenous distribution (Figure 2c, Figure S5, Supporting Information) of the components with atomic ratio as expected in $\mathrm{MnPX}_{3}$ (Figure S6, Supporting Information). Further crystallographic information can be collected from high resolution transmission electron microscope (HRTEM) and selected area electron diffraction (SAED) patterns. Figure $2 \mathrm{~d}$ shows a clear crystal fringes with lattice spacing of 0.3 and $0.51 \mathrm{~nm}$ which are indexed to the crystal planes of (-201) and (110), respectively. Complementary information can be elucidated from SAED pattern depicted in
Figure 2e where well-defined diffraction spots are clearly visualized corroborating the presence of characteristic planes for monoclinic $\mathrm{MnPS}_{3}$ (PDF\#78-0495). Figure 2f,g depicts the corresponding results for $\mathrm{MnPSe}_{3}$. From the HRTEM image, it is apparent that the lattice spacing of $0.371,0.208$, and $0.288 \mathrm{~nm}$ can be related to the crystal planes of (104), (211), and (113), respectively. Moreover, the SAED result unequivocally reveals a pure hexagonal $\mathrm{MnPSe}_{3}$ with crystal planes consistent with XRD pattern (PDF\# 89-1414). Beside structural identification, we carried out characterization with Raman spectroscopy to correlate the finger print peaks due to polarization driven modes of vibrations (Figure 3a,b). Being archetypal to most members of layered $\mathrm{MPX}_{3},{ }^{[21]}$ a band bellow $150 \mathrm{~cm}^{-1}$ is attributed to cation vibrations and a small peak representing $E_{\mathrm{u}}$ mode of vibration is apparent in both $\mathrm{MnPS}_{3}$ and $\mathrm{MnPSe}_{3}$ at around $155 \mathrm{~cm}^{-1}$.[22] As can be seen in Figure $3 \mathrm{a}$, the Raman modes originating from $\mathrm{P}_{2} \mathrm{~S}_{6}$ units with $\mathrm{D}_{3 \mathrm{~d}}$ symmetry (center of inversion along $\mathrm{PS}_{3}$ groups) are identified as $\mathrm{A}_{1 \mathrm{~g}}\left(245.9,381.8\right.$, and $\left.491 \mathrm{~cm}^{-1}\right)$ and $E_{\mathrm{g}}\left(225.5\right.$ and $\left.581 \mathrm{~cm}^{-1}\right) .{ }^{[23]}$ The out-of plane vibrations of $\mathrm{P}_{2} \mathrm{~S}_{6}$ entities (with P-P dimers) that are characterized by the opposite movement of $\mathrm{S}_{3} \mathrm{P}-\mathrm{PS}_{3}$ units can be ascertained from the strongly polarized $\mathrm{A}_{1 \mathrm{~g}}$ mode appearing at $381.8 \mathrm{~cm}^{-1}$.24] Prominent modes due to the $\mathrm{P}-\mathrm{S}$ bond symmetric stretching vibration are also corroborated from the peaks of Raman spectrum at 245.9 and $491 \mathrm{~cm}^{-1}$. Moreover, those peaks appearing at 274 and $581 \mathrm{~cm}^{-1}$ are in response to the in-plane vibrations of $\mathrm{P}_{2} \mathrm{~S}_{6}$ unit and represented by $E_{\mathrm{g}}$ mode. In the case of $\mathrm{MnPSe}_{3}$, belonging to hexagonal system, it possesses noncentrosymmetric $\mathrm{PSe}_{3}$ units and hence do not exhibit center of inversion. Another compelling remark can be made via comparison of the
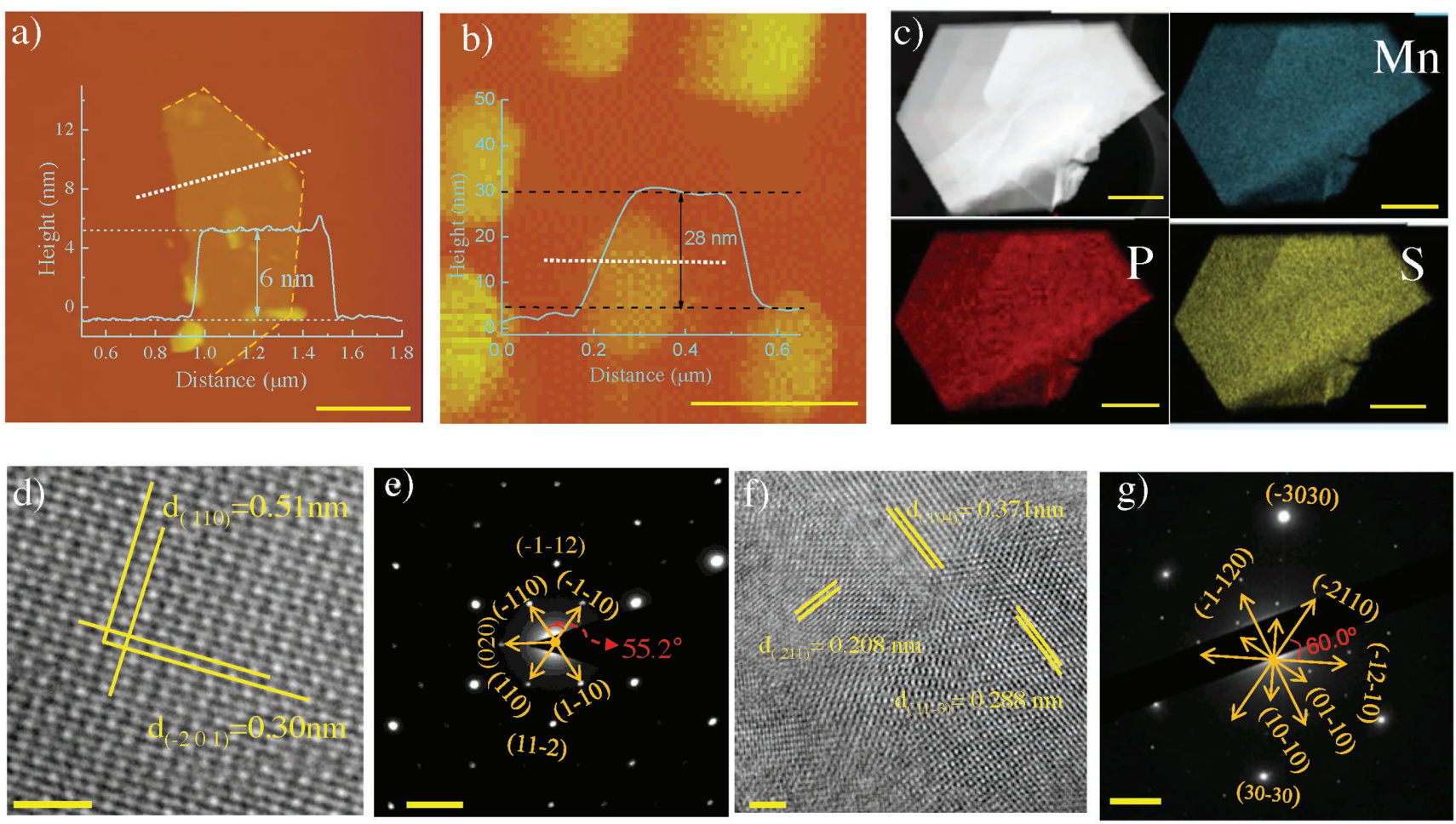

Figure 2. AFM images of a) $\mathrm{MnPS}_{3}$ and b) $\mathrm{MnPSe}_{3}$. EDX elemental mapping showing the uniform distribution of elements in c) $\mathrm{MnPS}_{3}$. $\mathrm{HRTEM}_{\mathrm{N}}$ and SAED spot pattern of $d, e) M_{n P S}$ and f,g) $M_{n P S e}$. Scale bars $(a, b)=0.5 \mu \mathrm{m} ;\left(c, d\right.$, and f) $=2 \mathrm{~nm} ;(e, g)=2 \mathrm{~nm}^{-1}$. 

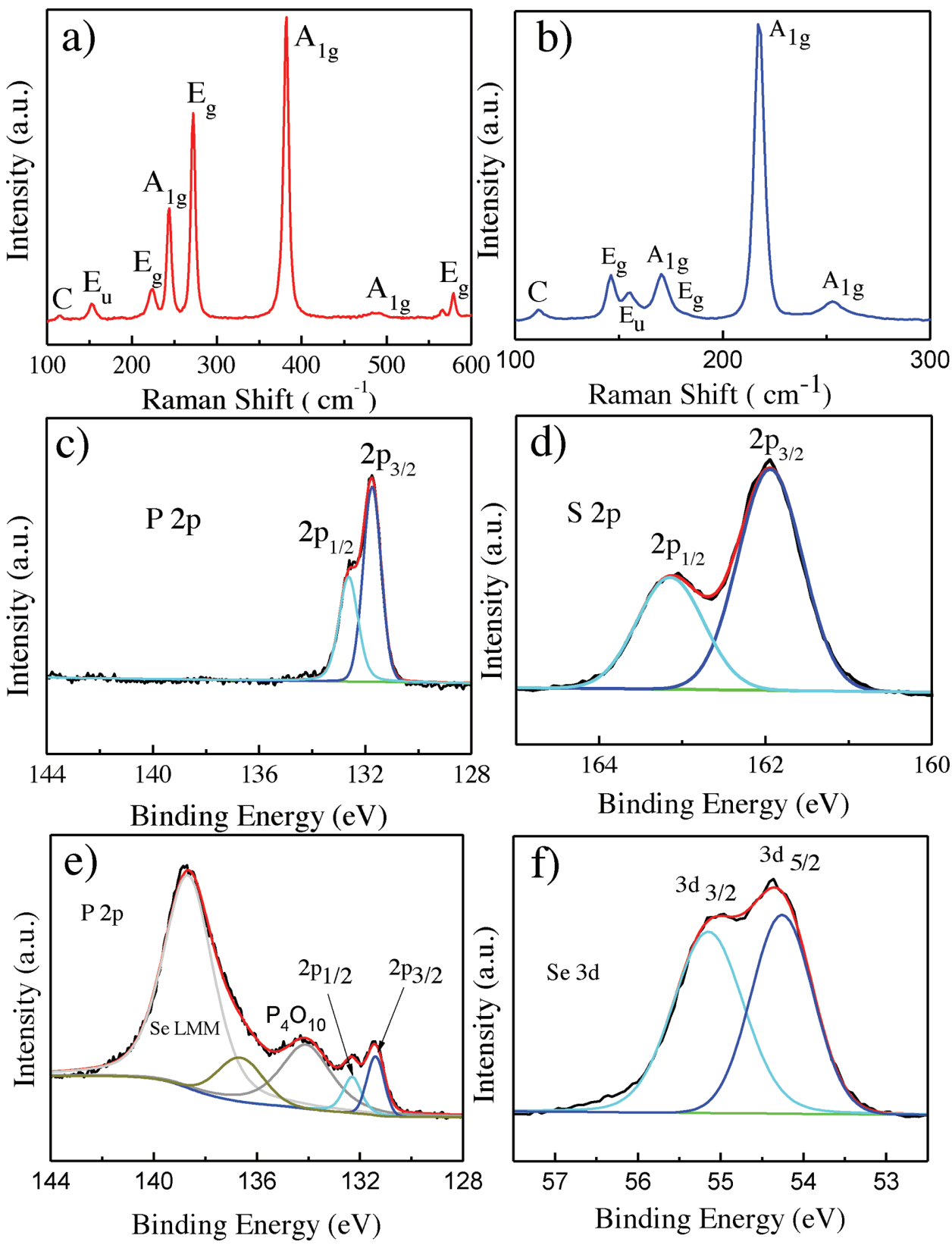

Figure 3. Raman spectra of a) $\mathrm{MnPS}_{3}$ and b) $\mathrm{MnPSe}_{3}$. High-resolution X-ray photoelectron spectra of c,d) P 2p, S 2p, and Se $3 d$ regions in MnPS and e,f) $\mathrm{MnPSe}_{3}$.

two Raman spectra of $\mathrm{MnPS}_{3}$ versus $\mathrm{MnPSe}_{3}$. The strongest peak at about $381 \mathrm{~cm}^{-1}$ in $\mathrm{MnPS}_{3}$ shifts to about $216 \mathrm{~cm}^{-1}$ in $\mathrm{MnPSe}_{3}$. As mentioned above, this peak is meant for the stretching vibration mode of $\mathrm{PX}_{3}$ units. The observed shift, therefore, tangibly corroborates the fact that chalcogen atoms are mainly involved in this motion no matter how the contribution from vibrational coupling be excluded. Analogous observation was made for $\mathrm{FePS}_{3}$ versus $\mathrm{FePSe}_{3} \cdot{ }^{[22]}$

Insight into the surface chemical states of the synthesized $\mathrm{MnPX}_{3}$ was gained from X-ray photoelectron spectroscopy (XPS). The $\mathrm{Mn}^{2+}$ state can be ascertained from the deconvoluted peaks of $\mathrm{Mn} \mathrm{P} 2 \mathrm{p}_{1 / 2}$ and $\mathrm{Mn} \mathrm{P} 2 \mathrm{p}_{3 / 2}$ at binding energies of 652 and $640 \mathrm{eV}$ along with shake-up satellite peak at $645 \mathrm{eV}$
(Figure S7, Supporting Information). Figure 3c-f shows the XPS spectra of $\mathrm{P}, \mathrm{S}$, and Se core levels from which the expected peaks for each elements as in the formation of $\mathrm{MnPX}_{3}$ are verified. The $S 2 p$ XPS spectrum shows well resolved peaks for $2 \mathrm{p}_{1 / 2}$ and $2 \mathrm{p}_{3 / 2}$ at binding energies of 161.9 and $163.1 \mathrm{eV}$, respectively, suggestive of $\mathrm{Mn}-\mathrm{S}$ and P-S entities. ${ }^{[23]}$ The Se $3 \mathrm{~d}$ spectrum (Figure 3f) depicts deconvoluted peaks at 54.22 and $55.14 \mathrm{eV}$ corresponding to Se $3 \mathrm{~d}_{3 / 2}$ and Se $3 \mathrm{~d}_{5 / 2}$, respectively, which are in good agreement with the reported values for $\mathrm{Se}$ 3d core level characteristics in $\mathrm{MnPSe}_{3} \cdot{ }^{[23]}$ In particular, it is conceivable to identify if chemically nonequivalent atoms of the same element are present in the investigated samples. It is tempting to describe that that there exists an obvious shift in 
the binding energy of $\mathrm{P}$ as seen in $\mathrm{MnPS}_{3}$ and $\mathrm{MnPSe}_{3}$. This might be associated to the slightly higher electronegativity of $\mathrm{S}$ as compared to Se. Given the fact that the core level binding energy of the central atom increases as the electronegativity of the atoms attached to it increases, $\mathrm{P} 2 \mathrm{p}$ in $\mathrm{MnPS}_{3}$ appears at 131.7 and 132.6 for $P 2 p_{1 / 2}$ and $P 2 p_{3 / 2}$, respectively, which are relatively at higher binding energies (by $\approx 0.3$ to $0.4 \mathrm{eV}$ ) than the case in $\mathrm{MnPSe}_{3}$. This corroborates the presence of nonequivalent atoms of the same element, $\mathrm{P}$, in $\mathrm{MnPS}_{3}$ and $\mathrm{MnPSe}_{3}$. Moreover, the additional peak at $138.7 \mathrm{eV}$ in Figure 3e can be attributed to the overlap of a Se Auger emission line and the P 2 p core level, which is in agreement with the observation made elsewhere. ${ }^{[24]}$

In order to know the light harvesting capacities of the assynthesized samples, we investigated their optical properties by UV-vis diffuse reflectance spectroscopy from which the absorption spectra are extracted and shown in Figure S8 (Supporting Information). It is obvious that the absorption edge of $\mathrm{MnPSe}_{3}$ shows a remarkable red shift signifying its enhanced light scavenging property as compared to $\mathrm{MnPS}_{3}$. Tauc plots shown in Figure $4 \mathrm{a}$ corroborate the fact that the synthesized materials are typical semiconductors with band gap energies $\left(E_{\mathrm{g}}\right)$ of 2.00 and $2.39 \mathrm{eV}$ for $\mathrm{MnPSe}_{3}$ and $\mathrm{MnPS}_{3}$, respectively. In order to determine the specific energy levels, we employed ultraviolet photoelectron spectroscopy (UPS) to get the absolute position of VB. By subtracting the width of He I UPS spectra from the system excitation energy of $21.22 \mathrm{eV}$ (Figure $4 \mathrm{~b}$ ), one can see that the VB levels of $\mathrm{MnPS}_{3}$ and $\mathrm{MnPSe}_{3}$ are at 1.15 versus NHE $(-5.59 \mathrm{eV}$ vs Vacuum) and 0.83 versus NHE $(-5.27 \mathrm{eV}$ vs Vacuum), respectively. Having these at hand, the energy level of $\mathrm{CB}$ can straightforwardly be calculated using the $E_{\mathrm{g}}$ value as 1.24 versus NHE (-3.2 eV vs Vacuum) and 1.17 versus NHE (-3.27 eV vs Vacuum) for $\mathrm{MnPS}_{3}$ and $\mathrm{MnPSe}_{3}$, respectively. Furthermore, we made a Mott-Schottky (M-S) analysis via measuring the space-charge layer capacitance over a scanned range of applied potentials ( 0.0 to $2.1 \mathrm{~V}$ vs saturated calomel electrode (SCE)) at 250 and $500 \mathrm{~Hz}$ (see the Experimental Section). Apart from being complementary to the above energy level calculations, $\mathrm{M}-\mathrm{S}$ plot can give information about the type of semiconductors under investigation. The negative slopes in Figure $4 c$ indicate that both $\mathrm{MnPS}_{3}$ and $\mathrm{MnPSe}_{3}$ are p-type semiconductors, in a good agreement with other reports. ${ }^{[14]}$ Meanwhile, the flat band potentials of $0.86 \mathrm{~V}$ versus reversible hydrogen electrode (RHE) (for $\mathrm{MnPS}_{3}$ ) and $0.55 \mathrm{~V}$ versus RHE (for $\mathrm{MnPSe}_{3}$ ) can be taken into account for estimating the energy level of VB; as VB is $0.3 \mathrm{~V}$ below the Fermi level for p-type semiconductors. ${ }^{[25]}$ Accordingly, one can see a very close proximity with the results obtained from UPS analysis.
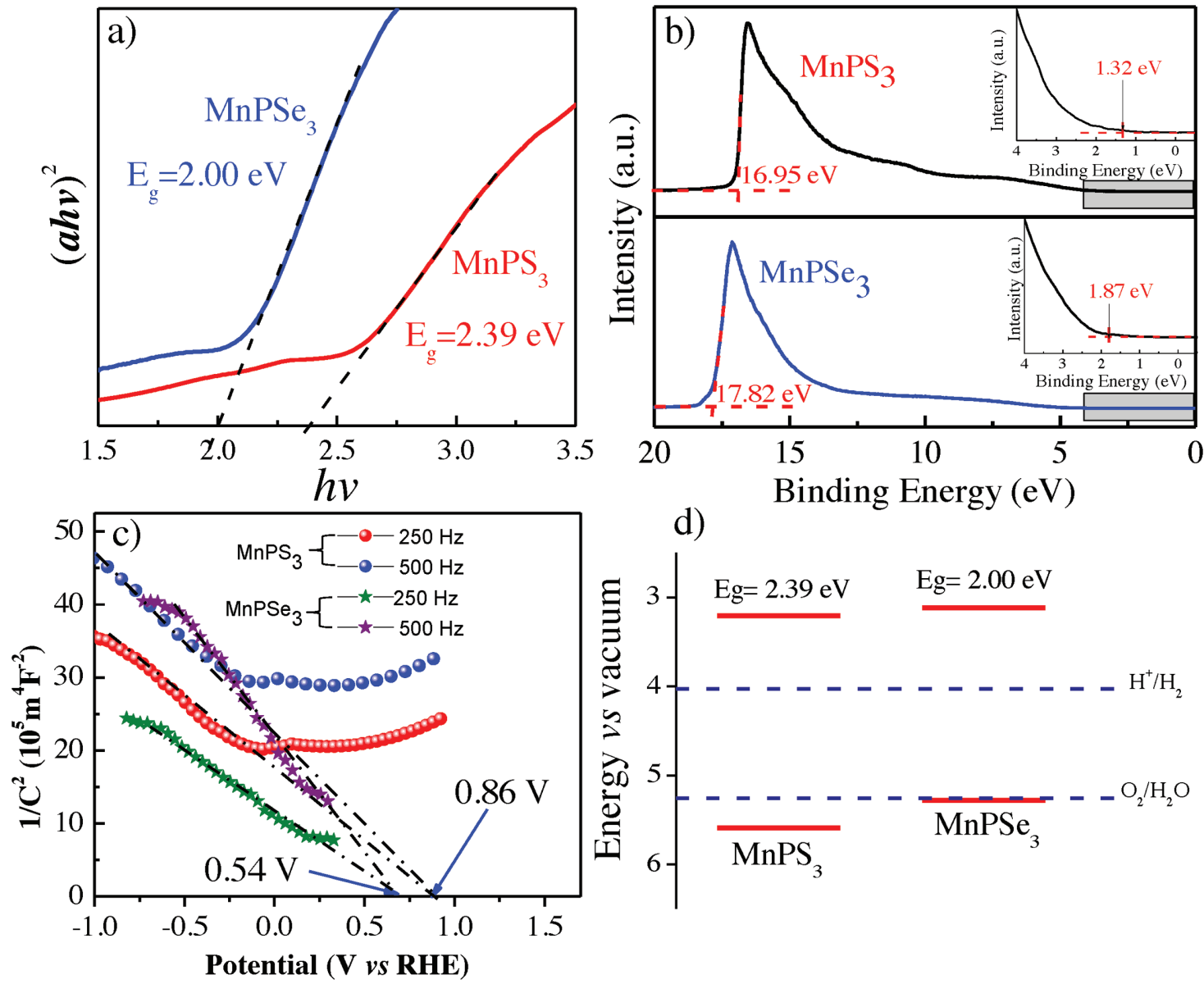

d)

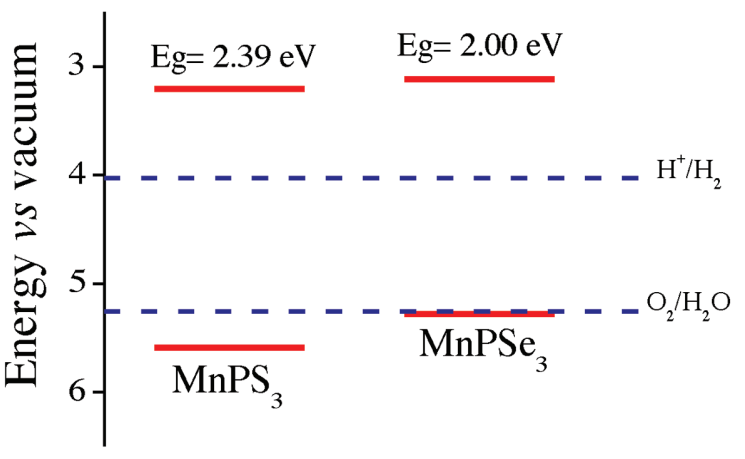

Figure 4. a) Tauc plots for the determination of band gaps. b) Ultraviolet photoelectron spectra. c) Mott-Schottky plots made at 250 and $500 \mathrm{~Hz}$. d) Schematic depicting the specific energy levels. 
Considering the energetically appropriate levels of $\mathrm{CB}$ for photocatalyzing the conversion of $\mathrm{H}^{+}$to $\mathrm{H}_{2}$ gas, we carried out sun light driven catalysis of water splitting using our obtained $\mathrm{MnPX}_{3}$ nanosheets. The photocatalytic performances were evaluated in pure water, without cocatalyst or sacrificial agents, under illumination of simulated solar light (AM $1.5 \mathrm{G})$. This experimental condition was followed to unequivocally explore the intrinsic property of the hitherto unexplored $\mathrm{MnPX}_{3}$ toward photocatalyzing water splitting. It is apparent from Figure 5 that both $\mathrm{MnPSe}_{3}$ and $\mathrm{MnPS}_{3}$ exhibit promising photocatalytic activities toward $\mathrm{H}_{2}$ gas production with a rate of 6.5 and $3.1 \mu \mathrm{mol} \mathrm{h}{ }^{-1}$, respectively. Moreover, the samples show appreciable stability in photocatalysis rendering a minor degradation after three cycles ( $6 \mathrm{~h}$ per each cycle). The rate for $\mathrm{MnPSe}_{3}\left(\mathrm{MnPS}_{3}\right)$ can reach to $43.5 \mu \mathrm{mol} \mathrm{h}-1\left(21.2 \mu \mathrm{mol} \mathrm{h}{ }^{-1}\right)$ in the presence of $\mathrm{Na}_{2} \mathrm{~S} / \mathrm{Na}_{2} \mathrm{SO}_{3}$ as hole scavenger (Figure $\mathrm{S} 9$, Supporting Information). The relatively better performance of $\mathrm{MnPSe}_{3}$ may be attributed to the following points: (1) The enhanced absorption in visible region that would account for generation of tremendous electrons at its conduction band, (2) The high carrier mobility, as it has been proved recently, ${ }^{[20]}$ which eases the accessibility of electrons to the incoming $\mathrm{H}^{+}$ and (3) given the fact that the constituents of the conduction bands are $\mathrm{P}_{2} \mathrm{X}_{6}{ }^{4-}$ cluster orbitals, the slight electronegativity difference between $\mathrm{S}$ and Se may endow an optimized condition for the $\mathrm{H}^{+}$adsorption and $\mathrm{H}_{2}$ desorption. However, we could not detect oxygen molecule in our system. This might be associated with the lack of sufficient active sites for promoting the kinetically sluggish $\mathrm{O}-\mathrm{O}$ covalent bond formation in oxygen evolution reaction (OER). ${ }^{[26]}$ Additionally, the energy level of $\mathrm{CB}$ is just slightly below the $\mathrm{O}_{2} / \mathrm{H}_{2} \mathrm{O}$ level that defies the evolution of oxygen and hence limited to only accumulating radical intermediates on the surface. As a matter of concept, we conducted a spin trapping experiment to trace surface hydroxyl radicals by using electron spin resonance spectroscopy (ESR). ESR spectra in Figure 5b show intensity ratio of 1:2:2:1 for both $\mathrm{MnPS}_{3}$ and $\mathrm{MnPSe}_{3}$ photocatalysts under the illumination of $\mathrm{Hg}$ light, illustrating characteristic features of hydroxyl radicals. ${ }^{[27]}$ The finding corroborates the fact that the photogenerated holes have oxidized the adsorbed water molecules to hydroxyl radicals on the surface of the catalysts. Thus, the rout toward $\mathrm{O}-\mathrm{O}$ covalent bond formation in OER is not completed under our experimental condition. This can be hastened by designing suitable cocatalyst that would assist the coupling of hydroxyl radicals and formation of superoxides to eventually result in the evolution of oxygen gas.

\section{Conclusion}

In conclusion, we report here a rationally designed novel route of synthesizing manganese-based transition metal phospho-trichalcogenides. Having optimized the CVD growth conditions, nanosheets of $\mathrm{MnPS}_{3}$ and $\mathrm{MnPSe}_{3}$ are successfully grown on flexible CF substrate. Following the theoretically speculated photocatalytic activity of direct band gap $\mathrm{MnPX}_{3}$ nanosheets, sunlight-driven catalytic activity is measured and promising performances are recorded. This work can ease the subsequent experimental efforts in this emerging family of 2D materials in the quest for the breakthroughs of water splitting catalysis and other optoelectronic devices.
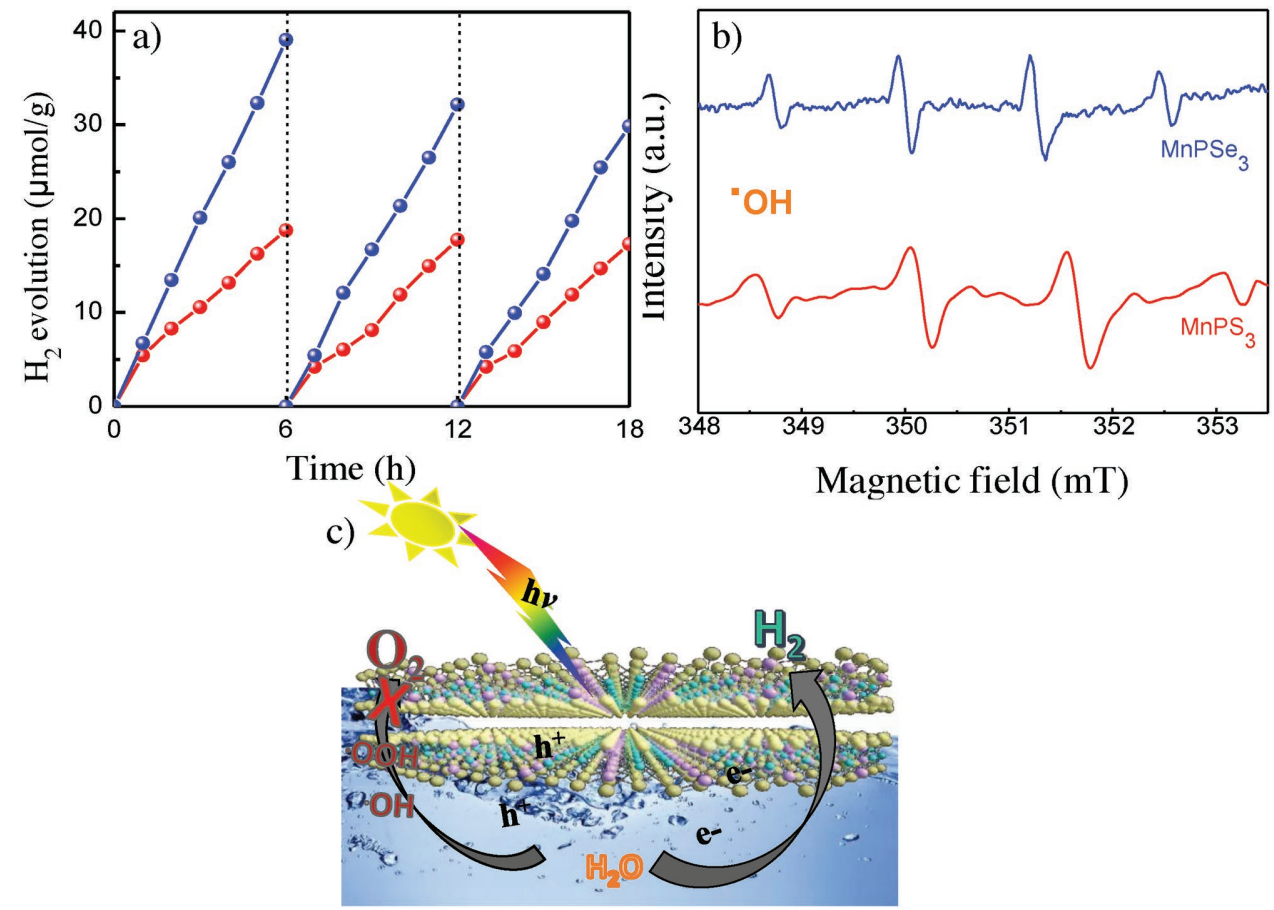

Figure 5. a) Photocatalytic $\mathrm{H}_{2}$ evolution rates under simulated solar light illumination along with stability tests of three cycles (6 h per cycle), red: $\mathrm{MnPS}_{3}$, blue: $\mathrm{MnPSe}_{3}$. b) ESR signals for DMPO-OH spin adduct. c) Schematics of water splitting to $\mathrm{H}_{2}$ gas and radical intermediates of oxide moieties revealing that the OER route is incomplete. 


\section{Experimental Section}

Synthesis of $\mathrm{MnO}_{2}$ Nanosheets on Carbon Fiber: Hydrothermal method was followed to synthesize $\mathrm{MnO}_{2}$ nanosheets with vertically standing architecture. A piece of CF was ultrasonically cleaned with acetone, ethanol, and ultrapure water before use. The cleaned CF substrate was acidified with $0.1 \mathrm{M} \mathrm{HNO}_{3}$ to functionalize the surface for dense growth of $\mathrm{MnO}_{2}$ nanosheets. The pretreated substrate was then rinsed in ethanol and water before being placed in Teflon-lined autoclave containing aqueous solution of $\mathrm{KMnO}_{4}(0.1 \mathrm{~g}$ in $50 \mathrm{~mL}$ of distilled water). Afterward, the content was sealed in iron cover and kept at $80^{\circ} \mathrm{C}$ for $12 \mathrm{~h}$. Eventually, the $\mathrm{MnO}_{2} / \mathrm{CF}$ was taken out, rinsed with ethanol and ultrapure water, and dried at $60^{\circ} \mathrm{C}$ before it was used as precursor in the subsequent CVD growth.

Synthesis of $\mathrm{MnPX}_{3}$ Nanosheets: $\mathrm{MnPX}_{3}(\mathrm{X}=\mathrm{S}$ or Se) nanosheets were facilely grown in a two zone chemical vapor deposition technique. The front thermal zone was loaded by a weighed amount of mixture of $\mathrm{P}$ and $\mathrm{S}$ (for $\mathrm{MnPS}_{3}$ ) or Se (for $\mathrm{MnPSe}_{3}$ ) powders whereas the hydrothermally grown $\mathrm{MnO}_{2} \mathrm{NS} / \mathrm{CF}$ was placed at the back zone. The system was flushed with $\mathrm{Ar}$ gas three times and pumped into a vacuum lower than $1 \mathrm{~Pa}$ in order to create oxygen free environment. Before starting the growth, the front zone was heated to $110^{\circ} \mathrm{C}$ for $10 \mathrm{~min}$ to create a thiophosphate/ selenophosphate atmosphere that would take the $\mathrm{P}$ and chalcogen vapor together during the reaction to the deposition zone. The temperature of the front zone was set to $280{ }^{\circ} \mathrm{C}\left(\mathrm{MnPS}_{3}\right)$ or $300{ }^{\circ} \mathrm{C}\left(\mathrm{MnPSe}_{3}\right)$ and that of the back zone was raised to $450^{\circ} \mathrm{C}\left(\mathrm{MnPS}_{3}\right)$ or $500^{\circ} \mathrm{C}\left(\mathrm{MnPSe}_{3}\right)$. The reaction was run for $2 \mathrm{~h}$ with $100 \mathrm{sccm}$ Ar gas flow, followed by naturally cooling of the furnace to room temperature. The obtained $\mathrm{MnPX}_{3} \mathrm{NS} / \mathrm{CF}$ was used as a photocatalyst without any pretreatment.

Characterizations: The morphology and crystal structure of the sample were investigated through AFM, field emission scanning electron microscopy (Hitachi S4800), and transmission electron microscopy (FEI Tecnai F20 TEM). The obtained sample, $\mathrm{MnPX}_{3} \mathrm{NS} / \mathrm{CF}$, was transferred to $\mathrm{SiO}_{2} / \mathrm{Si}$ substrate and ultrathin $\mathrm{Cu}$ grid for being visualized by AFM and TEM, respectively. XRD (D/MAX-TTRIII (CBO) diffractometer) was employed to acquire $\mathrm{X}$-ray diffractograms using $\mathrm{Cu} \mathrm{K} \alpha$ radiation. Elemental mapping images were obtained from STEM-EDX equipped on TEM-2100F (200 kV). Information about Raman spectra was gathered from confocal microscope-based Raman spectrometer (Renishaw InVia) in ambient air environment with an excitation laser line of $532 \mathrm{~nm}$. XPS spectra were collected from ESCALAB $250 \mathrm{Xi}$ (system of Thermo Scientific) with analysis chamber of $1.5 \times 10^{-9} \mathrm{mbar}$ and the $X$-ray spot of $500 \mu \mathrm{m}$. The base pressure in the analysis chamber was about $3 \times 10^{-9}$ mbar. Room temperature UV-Vis diffuse reflectance spectra were recorded using a Lambda 750 spectrophotometer in the wavelength range of 400-1000 nm. Ultraviolet photoelectron spectroscopy (Thermo Scientific ESCALab 250Xi) was used to estimate the valance band energy of MnPX3 hexagonal nanosheets. The gas discharge lamp was employed with the admitted helium gas ( $\mathrm{Hel}(21.22 \mathrm{eV}$ ) emission line). During analysis, the helium pressure inside the chamber was about $2 \mathrm{E}-8$ mbar. The data were collected with $-10 \mathrm{~V}$ bias.

Electrochemical Test: The flat band potential, as an estimation of band edge position, was measured via a typical three electrode system in $0.1 \mathrm{M} \mathrm{Na}_{2} \mathrm{SO}_{4}$ aqueous solution ( $\mathrm{pH}=6.57$ ). SCE, Pt wire, and the as-grown $\mathrm{MnPX}_{3} / \mathrm{CF}\left(\approx 1 \mathrm{~cm}^{2}\right)$ were used as reference, counter, and working electrodes, respectively. The required data for Mott-Schottky (M-S) analysis were collected via measuring the spacecharge layer capacitance over a scanned range of applied potentials $(0.0$ to $2.1 \mathrm{~V})$ at 250 and $500 \mathrm{~Hz}$ using Princeton Applied Research Potentiostat/Galvanostat VersaSTAT3 electrochemical analyzer. The recorded potentials were referenced to $\mathrm{RHE}$ according to $\mathrm{E}$ (vS RHE) = $\mathrm{E}$ (vs SCE) $+0.242 \mathrm{~V}+0.059 \times \mathrm{pH}$.

Photocatalytic Measurement: The photocatalytic water splitting reaction was carried out under simulated solar energy (AM 1.5G) illumination in a gas closed circulation system with a top-irradiation type reactor (quartz cell). Typically, the grown $\mathrm{MnPX}_{3} / \mathrm{CF}$ (mass loading $\approx 20 \mathrm{mg}$ ) was placed in a reactor containing $100 \mathrm{~mL}$ of ultrapure water. Before the reaction, the closed reactor system was degassed until $\mathrm{O}_{2}$ and $\mathrm{N}_{2}$ could not be detected. After starting irradiation, the evolving gas was quantified with a thermal conductivity detector TCD-based gas chromatograph (GC7900, Shimadzu, Japan, TCD, argon as a carrier gas and $5 \AA$ molecular sieve column) within each one hour of the illuminations. A base-line was calibrated by using the carrier Ar gas for each test before illumination.

Hydroxyl Radical Detection: Electron spin resonance spectrometer was used to detect water oxidation intermediate during photocatalysis. Since radical oxide moieties are short-lived in nature, 5,5-Dimethyl-1-pyrroline $\mathrm{N}$-oxide (DMPO) as a spin trapping agent was employed to easily detect hydroxyl radicals. In a typical experiment, the photocatalyst was immersed in $200 \mu \mathrm{L}$ distilled water and irradiated for $10 \mathrm{~min}$ followed by the addition of $50 \mu \mathrm{L}$ DMPO. Eventually, the ESR spectra were collected using Bruker E500 electron spin resonance spectrometer.

\section{Supporting Information}

Supporting Information is available from the Wiley Online Library or from the author.

\section{Acknowledgements}

T.S. and F.M.W. contributed equally to this work. This work was supported by Ministry of Science and Technology of China (No. 2016YFA0200700), National Natural Science Foundation of China (Nos. 61625401, 61474033, and 61574050), Strategic Priority Research Program of the Chinese Academy of Sciences (Grant No. XDA09040201), and CAS Key Laboratory of Nanosystem and Hierarchical Fabrication. The authors also gratefully acknowledge the support of Youth Innovation Promotion Association CAS and CAS-TWAS president's fellowship.

\section{Conflict of Interest}

The authors declare no conflict of interest.

\section{Keywords}

2D materials, chemical vapor deposition, manganese phosphorus trichalcogenides, photocatalysis, water splitting

Received: January 22, 2018

Revised: February 10, 2018 Published online:

[1] J. A. Turner, Science 2004, 305, 5686

[2] a) M. Chhowalla, H. S. Shin, G. Eda, L.-J. Li, K. P. Loh, H. Zhang, Nat. Chem. 2013, 5, 263; b) H. Zhang, ACS Nano 2015, 9, 9451; c) M. Chhowalla, Z. Liu, H. Zhang, Chem. Soc. Rev. 2015, 44, 2584; d) C. Tan, H. Zhang, Chem. Soc. Rev. 2015, 44, 2713; e) D. Deng, K. S. Novoselov, Q. Fu, N. Zheng, Z. Tian, X. Bao, Nat. Nanotechnol. 2016, 11, 218; f) W. Zhang, K. Zhou, Small 2017, 13, 1700806.

[3] a) J. Hu, C. Zhang, X. Meng, H. Lin, C. Hu, X. Long, S. Yang, J. Mater. Chem. A 2017, 5, 5995; b) F. Wang, T. A. Shifa, X. Zhan, Y. Huang, K. Liu, Z. Cheng, C. Jiang, J. He, Nanoscale 2015, 7, 19764; c) J. D. Benck, T. R. Hellstern, J. Kibsgaard, P. Chakthranont, T. F. Jaramillo, ACS Catal. 2014, 4, 3957.

[4] R. Ye, P. del Angel-Vicente, Y. Liu, M. J. Arellano-Jimenez, Z. Peng, T. Wang, Y. Li, B. I. Yakobson, S.-H. Wei, M. J. Yacaman, J. M. Tour, Adv. Mater. 2016, 28, 1427. 
[5] T. A. Shifa, F. Wang, K. Liu, Z. Cheng, K. Xu, Z. Wang, X. Zhan, C. Jiang, J. He, Small 2017, 13, 1603706.

[6] C. Ouyang, X. Wang, S. Wang, Chem. Commun. 2015, 51, 14160.

[7] M. Cabán-Acevedo, M. L. Stone, J. R. Schmidt, J. G. Thomas, Q. Ding, H.-C. Chang, M.-L. Tsai, J.-H. He, S. Jin, Nat. Mater. 2015, 14,1245

[8] W. Liu, E. Hu, H. Jiang, Y. Xiang, Z. Weng, M. Li, Q. Fan, X. Yu, E. I. Altman, H. Wang, Nat. Commun. 2016, 7, 10771.

[9] J. Kibsgaard, T. F. Jaramillo, Angew. Chem., Int. Ed. 2014, 53, 14433.

[10] Y. Shi, B. Zhang, Chem. Soc. Rev. 2016, 45, 1529.

[11] Y. Pan, Y. Liu, J. Zhao, K. Yang, J. Liang, D. Liu, W. Hu, D. Liu, Y. Liu, C. Liu, J. Mater. Chem. A 2015, 3, 1656.

[12] P. Xiao, M. A. Sk, L. Thia, X. Ge, R. J. Lim, J.-Y. Wang, K. H. Lim, X. Wang, Energy Environ. Sci. 2014, 7, 2624.

[13] J. F. Callejas, C. G. Read, E. J. Popczun, J. M. McEnaney, R. E. Schaak, Chem. Mater. 2015, 27, 3769.

[14] a) V. Grasso, F. Neri, S. Santangelo, L. Silipigni, M. Piacentini, J. Phys.: Condens. Matter 1989, 1, 3337; b) A. Carnabuci, V. Grasso, L. Silipigni, G. Salvato, J. Appl. Phys. 2001, 90, 4526.

[15] K.-z. Du, X.-z. Wang, Y. Liu, P. Hu, M. I. B. Utama, C. K. Gan, Q. Xiong, C. Kloc, ACS Nano 2016, 10, 1738.

[16] M. A. Susner, M. Chyasnavichyus, M. A. McGuire, P. Ganesh, P. Maksymovych, Adv. Mater. 2017, 29, 1602852.

[17] a) R. Nitsche, P. Wild, Mater. Res. Bull. 1970, 5, 419; b) B. E. Taylor, J. Steger, A. Wold, J. Solid State Chem. 1973, 7, 461; c) S. Hiroshi, Y. Takashi, S. Toshihiko, N. Satoru, H. Tooru, N. Saburo, Chem. Lett. 1997, 26, 1101.

[18] a) J. Shi, X. Wang, S. Zhang, L. Xiao, Y. Huan, Y. Gong, Z. Zhang, Y. Li, X. Zhou, M. Hong, Q. Fang, Q. Zhang, X. Liu, L. Gu, Z. Liu, Y. Zhang, Nat. Commun. 2017, 8, 958; b) Y.-H. Lee, X.-Q. Zhang, W. Zhang, M.-T. Chang, C.-T. Lin, K.-D. Chang, Y.-C. Yu,
J. T.-W. Wang, C.-S. Chang, L.-J. Li, T.-W. Lin, Adv. Mater. 2012, 24, 2320 .

[19] F. Wang, T. A. Shifa, P. He, Z. Cheng, J. Chu, Y. Liu, Z. Wang, F. Wang, Y. Wen, L. Liang, J. He, Nano Energy 2017, 40, 673.

[20] X. Zhang, X. Zhao, D. Wu, Y. Jing, Z. Zhou, Adv. Sci. 2016, 3, 1600062 .

[21] a) T. Senkine, M. Jouanne, C. Julien, M. Balkanski, Mater. Sci. Eng. 1989, 3, 91; b) M. Balkanski, M. Jouanne, G. Ouvrard, M. Scagliotti, J. Phys. C: Solid State Phys. 1987, 20, 4397.

[22] M. Scagliotti, M. Jouanne, M. Balkanski, G. Ouvrard, G. Benedek, Phys. Rev. B 1987, 35, 7097

[23] a) M. Hangyo, S. Nakashima, A. Mitsuishi, K. Kurosawa, S. Saito, Solid State Commun. 1988, 65, 419; b) R. Gusmão, Z. Sofer D. Sedmidubský, Š. Huber, M. Pumera, ACS Catal. 2017, 7, 8159; c) G. Long, T. Zhang, X. Cai, J. Hu, C.-w. Cho, S. Xu, J. Shen, Z. Wu, T. Han, J. Lin, J. Wang, Y. Cai, R. Lortz, Z. Mao, N. Wang, ACS Nano 2017, 11, 11330; d) L. Silipigni, G. Di Marco, G. Salvato, V. Grasso, Appl. Surf. Sci. 2005, 252, 1998.

[24] Y. Hou, M. Qiu, T. Zhang, X. Zhuang, C.-S. Kim, C. Yuan, X. Feng, Adv. Mater. 2017, 29, 1701589.

[25] L. Liao, Q. Zhang, Z. Su, Z. Zhao, Y. Wang, Y. Li, X. Lu, D. Wei, G. Feng, Q. Yu, X. Cai, J. Zhao, Z. Ren, H. Fang, F. RoblesHernandez, S. Baldelli, J. Bao, Nat. Nanotechnol. 2013, 9, 69.

[26] a) R. Li, F. Zhang, D. Wang, J. Yang, M. Li, J. Zhu, X. Zhou, H. Han, C. Li, Nat. Commun. 2013, 4, 1432; b) Q. Yuan, D. Liu, N. Zhang, W. Ye, H. Ju, L. Shi, R. Long, J. Zhu, Y. Xiong, Angew. Chem., Int. Ed. 2017, 129, 4270.

[27] a) Y. Nakabayashi, M. Nishikawa, N. Saito, C. Terashima, A. Fujishima, J. Phys. Chem. C 2017, 121, 25624; b) R. Shi, H.-F. Ye, F. Liang, Z. Wang, K. Li, Y. Weng, Z. Lin, W.-F. Fu, C.-M. Che, Y. Chen, Adv. Mater. 2018, 30, 1705941. 